\title{
On the Ulam type stability of several types of quadratic fuzzy set-valued functional equations
}

\author{
Yonghong Shen ${ }^{1,2^{*}}$, Wei Chen ${ }^{3}$ and Yaoyao Lan ${ }^{4}$
}

\section{"Correspondence:}

shenyonghong2008@hotmail.com

'School of Mathematics and

Statistics, Tianshui Normal

University, Tianshui, 741001, P.R. China

${ }^{2}$ School of Mathematics and Statistics, Beijing Institute of Technology, Beijing, 100081, P.R. China

Full list of author information is available at the end of the article

\begin{abstract}
Let $Y$ be a real separable Banach space and $\left(\mathcal{F}_{K C}(Y), d_{\infty}\right)$ be the space of all normal fuzzy convex and upper semicontinuous fuzzy sets with compact support in $Y$, where $d_{\infty}$ stands for the supremum metric in $\mathcal{F}_{K C}(Y)$. In the present paper, several types of quadratic fuzzy set-valued functional equations are introduced based on the space mentioned above. We prove the Hyers-Ulam stability of the standard quadratic fuzzy set-valued functional equation by using the fixed point technique. Simultaneously, we also establish some Ulam type stability results of the Deeba and Appolonius type fuzzy set-valued functional equations by employing the direct method, respectively. The stability results of the corresponding single-valued and set-valued functional equations acting as special cases will be included in our results.
\end{abstract}

Keywords: Ulam type stability; Hausdorff separation; supremum metric; quadratic fuzzy set-valued functional equation

\section{Introduction}

Nowadays, the Ulam type stability is gradually becoming one of the most active research topics in the theory of functional equations. The study of such stability problems of functional equations originated from a question of Ulam [1] concerning the stability of group homomorphisms, i.e.:

Let $G_{1}$ be a group and let $G_{2}$ be a metric group with the metric $d(\cdot, \cdot)$. Given $\epsilon>0$, does there exist a $\delta>0$ such that if a function $h: G_{1} \rightarrow G_{2}$ satisfies the inequality $d(h(x y), h(x) h(y))<\delta$ for all $x, y \in G_{1}$, then there is a homomorphism $H: G_{1} \rightarrow G_{2}$ with $d(h(x), H(x))<\epsilon$ for all $x \in G_{1}$ ?

Afterwards, Hyers [2] gave a first affirmative partial answer to the question of Ulam for Banach spaces. Later, this result was generalized by Aoki [3] for additive mappings and independently by Rassias [4] for linear mappings by considering an unbounded Cauchy deference. In 1994, a further generalization of T.M. Rassias theorem was obtained by Găvruța [5]. Since then, the Ulam type stability problems for different types of functional equations in various abstract spaces have been widely and extensively studied. For more details, we refer the readers to [6-10]. Moreover, it is worth noting that the study of Ulam stability 
for some quadratic functional equations is important and interesting. For instance,

$$
\begin{aligned}
& f(x+y)+f(x-y)=2 f(x)+2 f(y) \quad \text { (Standard type), } \\
& f(x+y+z)+f(x)+f(y)+f(z)=f(x+y)+f(y+z)+f(z+x) \quad \text { (Deeba type), } \\
& f(x-z)+f(y-z)=\frac{1}{2} f(x-y)+2 f\left(z-\frac{x+y}{2}\right) \quad \text { (Appolonius type). }
\end{aligned}
$$

It should be noted that the function $f(x)=x^{2}$ is a solution of the three equations above. Naturally, we call these equations quadratic functional equations and every solution a quadratic function (mapping). In the case of single-valued equations, the corresponding Ulam type stability of these equations have been investigated by Czerwik [11], Kim [12] and Jung [13], Jun and Kim [14], respectively.

In 2008, Mirmostafaee and Moslehian [15] initiated the study of the Ulam stability problems of functional equations in fuzzy setting. More precisely, they discussed the Ulam stability of Cauchy type and then in the same year, together with Mirzavaziri [16], Jensen type functional equations in a complete fuzzy normed linear space. Thereafter, the stability of various functional equations in a fuzzy abstract space has been investigated by various authors [17-25].

Summing up the above, the Ulam type stability problem is whether, for a given mapping which satisfies approximately a functional equation (or which satisfies a functional inequality), there exists an exact solution of the corresponding functional equation such that the preceding mapping is sufficiently close to this solution. Usually, an appropriate metric associated with the corresponding space is chosen to characterize the error in functional inequalities. Certainly, a fuzzy metric is adopted in fuzzy environment. Unlike previous studies, Lu and Park [26] first studied the stability of two types of additive set-valued functional equations, in which the inclusion relation is employed to measure the degree of approximation. Soon after, Park et al. [27] further investigated the stability of quadratic, cubic and quartic set-valued functional equations. However, the disadvantage is that the obtained exact solution of these set-valued functional equations is just single-valued mapping. Recently, Kenary et al. [28] studied the stability of several types of set-valued functional equations by using the fixed point method. It should be pointed out that the same skill has been applied to solve the Ulam stability problems of single-valued and set-valued functional equations, respectively.

The main objective of this paper is to further extend and establish some new Ulam type stability results of the quadratic functional equations mentioned above, in which the quadratic mapping is assumed to be a fuzzy set-valued mapping. More specifically, the standard quadratic, Deeba and Appolonius type fuzzy set-valued functional equations. In the mean time, the supremum metric will be used to estimate the error in fuzzy set-valued functional inequalities.

\section{Preliminaries}

In this section, we present some related concepts and results which are mainly derived from [29-32].

Let $Y$ be a real separable Banach space with the norm $\|\cdot\|_{Y}$. We denote by $\mathcal{K}(Y)$ and $\mathcal{K}_{C}(Y)$ the set of all nonempty compact subsets of $Y$ and the set of all nonempty compact convex subsets of $Y$, respectively. 
Let $A$ and $B$ be two nonempty subsets of $Y$ and let $\lambda \in \mathbb{R}$. The (Minkowski) addition and scalar multiplication can be defined by

$$
A+B=\{a+b \mid a \in A, b \in B\}
$$

and

$$
\lambda A=\{\lambda a \mid a \in A\} .
$$

Notice that the sets $\mathcal{K}(Y)$ and $\mathcal{K}_{C}(Y)$ are closed under the operations of addition and scalar multiplication. In fact, these two operations induce a linear structure on $\mathcal{K}(Y)$ and $\mathcal{K}_{C}(Y)$ with zero element $\{0\}$, respectively. It should be noted that this linear structure is just a cone rather than a vector space because, in general, $A+(-1) A \neq\{0\}$. Moreover, for all $\lambda, \mu \in \mathbb{R}$, it follows that

$$
\lambda(A+B)=\lambda A+\lambda B, \quad(\lambda+\mu) A \subseteq \lambda A+\mu A .
$$

Note that if $A$ is convex and $\lambda \mu \geq 0$, then $(\lambda+\mu) A=\lambda A+\mu A$.

Define the Hausdorff separation of $B$ from $A$ by

$$
d_{H}^{*}(B, A)=\inf \left\{\epsilon>0 \mid B \subseteq A+\epsilon \overline{S_{1}}\right\},
$$

where $\overline{S_{1}}$ denotes the closed unit ball in $Y$, i.e., $\overline{S_{1}}=\left\{y \in Y \mid\|y\|_{Y} \leq 1\right\}$. The Hausdorff separation of $A$ from $B$ can also be defined similarly.

Based on these two types of separations, the Hausdorff distance between nonempty subsets $A$ and $B$ is defined by

$$
d_{H}(A, B)=\max \left\{d_{H}^{*}(A, B), d_{H}^{*}(B, A)\right\} .
$$

In general, the Hausdorff distance has the following properties:

(i) $d_{H}(\lambda A, \lambda B)=|\lambda| d_{H}(A, B)$ for all $\lambda \in \mathbb{R}$;

(ii) $d_{H}(A+C, B+C)=d_{H}(A, B)$;

(iii) $d_{H}(A+C, B+D) \leq d_{H}(A, B)+d_{H}(C, D)$ for all $A, B, D \in \mathcal{K}(Y)$ and $C \in \mathcal{K}_{C}(Y)$.

If we restrict our attention to the nonemtpy closed subsets $\mathcal{C}(Y)$ of $Y$, then one can obtain the fact from [29] that $\left(\mathcal{C}(Y), d_{H}\right)$ is a complete metric space. Clearly, $\mathcal{K}(Y)$ and $\mathcal{K}_{C}(Y)$ are closed subsets of $\mathcal{C}(Y)$. Hence, $\left(\mathcal{K}(Y), d_{H}\right)$ and $\left(\mathcal{K}_{C}(Y), d_{H}\right)$ are also complete metric spaces.

In the 1990s, the concept of the Banach space-valued fuzzy sets was introduced by Inoue [31], who extended the usual fuzzy sets defined on $\mathbb{R}$ or $\mathbb{R}^{n}$. In other words, the base space of a fuzzy set is replaced by a more general Banach space.

A fuzzy set defined on $Y$ is a mapping $u: Y \rightarrow[0,1]$. We denote by $\mathcal{F}(Y)$ the set of all fuzzy sets defined on $Y$. Let $\mathcal{F}_{K}(Y)$ denote the class of fuzzy sets $u: Y \rightarrow[0,1]$ with the following properties:

(i) $u$ is normal, i.e., there exists $y_{0} \in Y$, such that $u\left(y_{0}\right)=1$;

(ii) $u$ is upper semicontinuous;

(iii) $[u]^{\alpha}=\{y \in Y \mid u(y) \geq \alpha\}$ is compact for each $\alpha \in(0,1]$;

(iv) $[u]^{0}=\overline{\bigcup_{\alpha \in(0,1]}[u]^{\alpha}}$ is a bounded subset of $Y$. 
In fact, the conditions (ii) and (iv) imply that $[u]^{0}$ is also compact. Moreover, we use the notation $\mathcal{F}_{K C}(Y)$ to denote the subspace of $\mathcal{F}(Y)$ whose members also satisfy

(v) $[u]^{\alpha}$ is convex for each $\alpha \in(0,1]$, that is to say, $u$ is fuzzy convex.

A linear structure can be defined in $\mathcal{F}(Y)$ in a similar way to fuzzy sets in $\mathbb{R}$ or $\mathbb{R}^{n}$, by

$$
\begin{aligned}
& (u \oplus v)(y)=\sup _{x+z=y} \min \{u(x), v(z)\}, \\
& (\gamma u)(y)= \begin{cases}u\left(\frac{y}{\gamma}\right), & \text { if } \gamma \neq 0, \\
I_{0}(y), & \text { if } \gamma=0\end{cases}
\end{aligned}
$$

for $u, v \in \mathcal{F}(Y)$ and $\gamma \in \mathbb{R}$, where $I_{0}(y)=0$ if $y \neq 0$ and $I_{0}(0)=1$. Then $\mathcal{F}(Y)$ is closed under these operations, and level setwise

$$
[u \oplus v]^{\alpha}=[u]^{\alpha}+[v]^{\alpha}, \quad[\lambda u]^{\alpha}=\lambda[u]^{\alpha}
$$

for each $\alpha \in[0,1]$ and $\lambda \in \mathbb{R}$. Similar to the closeness of $\mathcal{K}_{C}(Y)$, it is easy to see that $\mathcal{F}_{K C}(Y)$ is also closed under these operations.

Based on the statement mentioned above, for any $u, v \in \mathcal{F}_{K C}(Y)$ and $\lambda, \mu \in \mathbb{R}$, the following equalities hold:

(i) $\lambda(u \oplus v)=\lambda u \oplus \lambda v$;

(ii) $\lambda(\mu u)=(\lambda \mu) u$;

(iii) $(\lambda+\mu) u=\lambda u \oplus \mu u$ for any $\lambda, \mu \geq 0$.

Therefore, it is easy to check that the set $\mathcal{F}_{K C}(Y)$ is just a cone defined on $Y$ rather than a vector space.

As a generalization of the Hausdorff metric $d_{H}$ in $\mathcal{K}(Y)$, the supremum metric is defined by

$$
d_{\infty}(u, v)=\sup _{\alpha \in(0,1]} d_{H}\left([u]^{\alpha},[v]^{\alpha}\right)
$$

where $u, v \in \mathcal{F}_{C}(Y)$.

Remark 1 Every ordinary crisp subset $A$ of $Y$ can be identified with the fuzzy set on $Y$ by its characteristic function $\chi_{A}: Y \rightarrow\{0,1\}$, that is with $\chi_{A}(y)=1$ if $y \in A$ and $\chi_{A}(y)=0$ if $y \notin A$. Therefore, if $A \in \mathcal{K}(Y)$ (or $A \in \mathcal{K}_{C}(Y)$ ), then $\chi_{A} \in \mathcal{F}_{C}(Y)$ (or $\mathcal{F}_{K C}(Y)$ ), and vice versa.

From Remark 1, for any $A, B \in \mathcal{K}(Y)$ (or $\mathcal{F}_{K C}(Y)$ ), it follows that

$$
d_{\infty}\left(\chi_{A}, \chi_{B}\right)=\sup _{\alpha \in(0,1]} d_{H}\left(\left[\chi_{A}\right]^{\alpha},\left[\chi_{B}\right]^{\alpha}\right)=d_{H}(A, B)
$$

Especially, if $A$ and $B$ are simplified into two singleton sets $\{a\}$ and $\{b\}$, respectively, then we can infer from equality $(1)$ that $d_{\infty}\left(\chi_{\{a\}}, \chi_{\{b\}}\right)=d(a, b)$, where $d$ denotes the usual metric between $a$ and $b$.

According to the properties of the Hausdorff metric, it can easily be shown that the supremum metric has the following properties: 
(i) $d_{\infty}(\lambda u, \lambda v)=\lambda d_{\infty}(u, v)$ for any $\lambda \geq 0$;

(ii) $d_{\infty}(u \oplus w, v \oplus w)=d_{\infty}(u, v)$;

(iii) $d_{\infty}\left(u \oplus v, u^{\prime} \oplus v^{\prime}\right) \leq d_{\infty}\left(u, u^{\prime}\right)+d_{\infty}\left(v, v^{\prime}\right)$ for all $u, v, w, u^{\prime}, v^{\prime} \in \mathcal{F}_{K C}(Y)$.

Remark 2 If we restrict our attention to the set $\mathcal{F}_{K C}(Y)$, we then can prove that $\left(\mathcal{F}_{K C}(Y)\right.$, $d_{\infty}$ ) is a complete metric space by using the method analogous to that used in [30, Proposition 7.2.3].

Here we recall a fixed point theorem in a complete generalized metric space which are useful in the next section.

Theorem 2.1 (Diaz and Margolis [33]) Let $(X, d)$ be a complete generalized metric space, i.e., one for which $d$ may assume infinite values. Suppose that $J: X \rightarrow X$ be a strictly contractive mapping with Lipschitz constant $L<1$. Then, for each given element $x \in X$, either

$$
d\left(J^{n} x, J^{n+1} x\right)=\infty
$$

for all $n \geq 0$ or there exists an $n_{0} \in \mathbb{N}$ such that

(i) $d\left(J^{n} x, J^{n+1} x\right)<\infty$ for all $n \geq n_{0}$;

(ii) the sequence $\left\{J^{n} x\right\}$ converges to a fixed point $y^{*}$ of $J$;

(iii) $y^{*}$ is the unique fixed point of $J$ in the set $Y=\left\{y \in X \mid d\left(J^{n_{0}} x, y\right)<\infty\right\}$;

(iv) $d\left(y, y^{*}\right) \leq \frac{1}{1-L} d(y, J y)$ for all $y \in Y$.

\section{Hyers-Ulam stability of the standard quadratic fuzzy set-valued functional equation}

In this section, we shall establish the Hyers-Ulam stability of the standard quadratic fuzzy set-valued functional equation by using the fixed point technique.

Definition 3.1 Let $X$ be a real vector space and let $f: X \rightarrow \mathcal{F}_{K C}(Y)$ be a fuzzy set-valued mapping. The quadratic fuzzy set-valued functional equation is defined by

$$
f(x+y) \oplus f(x-y)=2 f(x) \oplus 2 f(y)
$$

for all $x, y \in X$, where the equal sign means that the both sides of (2) (two fuzzy sets) are equal pointwise. Every solution of (2) is called a standard quadratic fuzzy set-valued mapping.

Example 1 Let $X=Y=\mathbb{R}$. Suppose that $f: \mathbb{R} \rightarrow \mathcal{F}_{K C}(Y)$ is a triangular fuzzy set-valued mapping, i.e., for every $t \in X, f(t)$ is a triangular fuzzy set in $\mathbb{R}$, which is defined by

$$
f(t)=\left(t^{2}-a t^{2}, t^{2}, t^{2}+b t^{2}\right), \quad t \in \mathbb{R}
$$

where $a, b$ are two nonnegative real numbers. From the definition of an $\alpha$-level set of a fuzzy set, it follows that

$$
[f(t)]^{\alpha}=\left[t^{2}-a t^{2}(1-\alpha), t^{2}+b t^{2}(1-\alpha)\right]
$$


for every $t \in \mathbb{R}$ and $\alpha \in[0,1]$. Thus, for every $\alpha \in[0,1]$, it can easily be verified that

$$
[f(x+y)]^{\alpha}+[f(x-y)]^{\alpha}=2[f(x)]^{\alpha}+2[f(y)]^{\alpha}
$$

for all $x, y \in X$. This implies that $f$ is a solution of (2) in $\mathbb{R}$.

Remark 3 Let $u_{0} \in \mathcal{F}_{K C}(Y)$ be a fixed fuzzy set. Accordingly, it is easy to check that $f(t)=$ $t^{2} u_{0}$ is also a solution of (2) by the same method as shown in Example 1.

Theorem 3.1 Let $j \in\{-1,1\}$ be fixed and let $\varphi: X \times X \rightarrow[0, \infty)$ be a function such that there exists a positive constant $L<1$ satisfying

$$
\varphi(x, y) \leq 4^{j} L \varphi\left(2^{-j} x, 2^{-j} y\right)
$$

for all $x, y \in X$. Assume that $f: X \rightarrow \mathcal{F}_{K C}(Y)$ is a mapping with $f(0)=I_{0}$ which satisfies

$$
d_{\infty}(f(x+y) \oplus f(x-y), 2 f(x) \oplus 2 f(y)) \leq \varphi(x, y)
$$

for all $x, y \in X$, then

$$
Q(x)=\lim _{n \rightarrow \infty} 4^{-j n} f\left(2^{j n} x\right)
$$

exists for each $x \in X$ and defines a unique quadratic fuzzy set-valued mapping $Q: X \rightarrow$ $\mathcal{F}_{K C}(Y)$ such that

$$
d_{\infty}(f(x), Q(x)) \leq \begin{cases}\frac{1}{4(1-L)} \varphi(x, x), & \text { if } j=1, \\ \frac{L}{4(1-L)} \varphi(x, x), & \text { if } j=-1\end{cases}
$$

for all $x \in X$.

Proof Putting $y=x$ in (4), we can obtain

$$
d_{\infty}\left(\frac{1}{4} f(2 x), f(x)\right) \leq \frac{1}{4} \varphi(x, x)
$$

for all $x \in X$, since $f(0)=I_{0}$ and $u \oplus I_{0}=u$ for any $u \in \mathcal{F}_{K C}(Y)$. Furthermore, it follows from (3) that

$$
d_{\infty}\left(f(x), 4 f\left(\frac{x}{2}\right)\right) \leq \varphi\left(\frac{x}{2}, \frac{x}{2}\right) \leq \frac{L}{4} \varphi(x, x)
$$

for all $x \in X$.

Consider the set $E=\left\{g \mid g: X \rightarrow \mathcal{F}_{K C}(Y), g(0)=I_{0}\right\}$ and introduce the generalized metric $D$ on $E$, which is defined by

$$
D(g, h)=\inf \left\{\mu \in(0, \infty) \mid d_{\infty}(g(x), h(x)) \leq \mu \varphi(x, x), \forall x \in X\right\}
$$


where, as usual, $\inf \emptyset=\infty$. In can easily be verified that $(E, D)$ is a complete generalized metric space (see [34], Theorem 2.4).

Now, we define an operator $J: E \rightarrow E$ by

$$
J g(x)=4^{-j} g\left(2^{j} x\right)
$$

for all $x \in X$.

Let $g, h$ be given such that $D(g, h)=\epsilon$. Then

$$
d_{\infty}(g(x), h(x)) \leq \epsilon \varphi(x, x)
$$

for all $x \in X$. Hence, we can obtain

$$
\begin{aligned}
d_{\infty}(J g(x), J h(x)) & =d_{\infty}\left(4^{-j} g\left(2^{j} x\right), 4^{-j} h\left(2^{j} x\right)\right) \\
& =4^{-j} d_{\infty}\left(g\left(2^{j} x\right), h\left(2^{j} x\right)\right) \\
& \leq \epsilon 4^{-j} \varphi\left(2^{j} x, 2^{j} x\right) \\
& \leq L \epsilon \varphi(x, x)
\end{aligned}
$$

for all $x \in X$. So $D(g, h)=\epsilon$ implies that $D(J g, J h) \leq L \epsilon$. This means that

$$
D(g, J h) \leq L D(g, h)
$$

for all $g, h \in E$. That is to say, $J$ is a strictly contractive self-mapping on $E$ with Lipschitz constant $L<1$.

Moreover, we can infer that from (6) and (7) that

$$
D(f, J f) \leq \begin{cases}\frac{1}{4}, & \text { if } j=1, \\ \frac{L}{4}, & \text { if } j=-1 .\end{cases}
$$

According to Theorem 2.1, there exists a mapping $Q: X \rightarrow \mathcal{F}_{K C}(Y)$ satisfying the following:

(i) $Q$ is a fixed point of $J$, i.e.,

$$
4^{j} Q(x)=Q\left(2^{j} x\right)
$$

for all $x \in X$. The mapping $Q$ is the unique fixed point of $J$ in the set

$$
M=\{g \in E \mid D(f, g)<\infty\},
$$

which implies that $Q$ is the unique mapping satisfying (9) such that there exists an $\eta \in(0,1)$ satisfying

$$
d_{\infty}(f(x), Q(x)) \leq \eta \varphi(x, x)
$$

for all $x \in X$. 
(ii) $D\left(J^{n} f, Q\right) \rightarrow 0$ as $n \rightarrow \infty$. This implies the equality

$$
\lim _{n \rightarrow \infty} 4^{-j n} g\left(2^{j n} x\right)=Q(x)
$$

for all $x \in X$.

(iii) $D(f, Q) \leq \frac{1}{1-L} D(f, J f)$, which implies the inequality

$$
D(f, Q) \leq \begin{cases}\frac{1}{4(1-L)}, & \text { if } j=1 \\ \frac{L}{4(1-L)}, & \text { if } j=-1\end{cases}
$$

This shows that the inequality (5) holds.

Replacing $x$ and $y$ by $2^{j n} x$ and $2^{j n} y$ in (4), we can infer that

$$
\begin{aligned}
& d_{\infty}\left(4^{-j n} f\left(2^{j n}(x+y)\right) \oplus 4^{-j n} f\left(2^{j n}(x-y)\right), 2 \cdot 4^{-j n} f\left(2^{j n} x\right) \oplus 2 \cdot 4^{-j n} f\left(2^{j n} y\right)\right) \\
& \leq 4^{-j n} \varphi\left(2^{j n} x, 2^{j n} y\right) \leq 4^{-j n} \cdot 4^{j n} L^{n} \varphi(x, y)=L^{n} \varphi(x, y),
\end{aligned}
$$

which tends to zero as $n \rightarrow \infty$ for all $x, y \in X$. Thus,

$$
Q(x+y) \oplus Q(x-y)=2 Q(x) \oplus 2 Q(y)
$$

for all $x, y \in X$ and therefore the mapping $Q: X \rightarrow \mathcal{F}_{K C}(Y)$ is a quadratic fuzzy set-valued mapping, as desired.

Corollary 3.2 Let $X$ be a real normed space and let $p, \theta$ be positive real numbers with $p \neq 2$. Assume that $f: X \rightarrow \mathcal{F}_{K C}(Y)$ is a mapping satisfying

$$
d_{\infty}(f(x+y) \oplus f(x-y), 2 f(x) \oplus 2 f(y)) \leq \theta\left(\|x\|^{p}+\|y\|^{p}\right)
$$

for all $x, y \in X$. Then

$$
Q(x)=\lim _{n \rightarrow \infty} 4^{-j n} f\left(2^{j n} x\right)
$$

exists for each $x \in X$ and defines a unique quadratic fuzzy set-valued mapping $Q: X \rightarrow$ $\mathcal{F}_{K C}(Y)$ such that

$$
d_{\infty}(f(x), Q(x)) \leq \begin{cases}\frac{\theta\|x\|^{p}}{2-2^{p-1}}, & \text { if } j=1, p<2, \\ \frac{\theta\|x\|^{p}}{2^{p-1}-2}, & \text { if } j=-1, p>2\end{cases}
$$

for all $x \in X$.

Proof In Theorem 3.1, let $\varphi(x, y)=\theta\left(\|x\|^{p}+\|y\|^{p}\right)$. Then we can choose $L=2^{j(p-2)}$ and we get the desired result.

Corollary 3.3 Let $X$ be a real normed space and let $p, \theta$ be positive real numbers with $p \neq 1$. Assume that $f: X \rightarrow \mathcal{F}_{K C}(Y)$ is a mapping satisfying

$$
d_{\infty}(f(x+y) \oplus f(x-y), 2 f(x) \oplus 2 f(y)) \leq \theta\|x\|^{p}\|y\|^{p}
$$


for all $x, y \in X$. Then

$$
Q(x)=\lim _{n \rightarrow \infty} 4^{-j n} f\left(2^{j n} x\right)
$$

exists for each $x \in X$ and defines a unique quadratic fuzzy set-valued mapping $Q: X \rightarrow$ $\mathcal{F}_{K C}(Y)$ such that

$$
d_{\infty}(f(x), Q(x)) \leq \begin{cases}\frac{\theta\|x\|^{2 p}}{4-4^{p}}, & \text { if } j=1, p<1, \\ \frac{\theta\|x\|^{2 p}}{4^{p}-4}, & \text { if } j=-1, p>1\end{cases}
$$

for all $x \in X$.

Proof In Theorem 3.1, let $\varphi(x, y)=\theta\|x\|^{p}\|y\|^{p}$. Then we can choose $L=4^{j(p-1)}$ and we get the desired result.

Corollary 3.4 Let $X$ be a real normed space and let $p, q, \theta$ be positive real numbers with $p+q \neq 2$. Assume that $f: X \rightarrow \mathcal{F}_{K C}(Y)$ is a mapping satisfying

$$
d_{\infty}(f(x+y) \oplus f(x-y), 2 f(x) \oplus 2 f(y)) \leq \theta\left(\|x\|^{p}\|y\|^{q}+\|x\|^{p+q}+\|y\|^{p+q}\right)
$$

for all $x, y \in X$. Then

$$
Q(x)=\lim _{n \rightarrow \infty} 4^{-j n} f\left(2^{j n} x\right)
$$

exists for each $x \in X$ and defines a unique quadratic fuzzy set-valued mapping $Q: X \rightarrow$ $\mathcal{F}_{K C}(Y)$ such that

$$
d_{\infty}(f(x), Q(x)) \leq \begin{cases}\frac{3 \theta\|x\|^{p+q}}{4-2^{p+q}}, & \text { if } j=1, p+q<2, \\ \frac{3 \theta\|x\|^{p+q}}{2^{p+q}-4}, & \text { if } j=-1, p+q>2\end{cases}
$$

for all $x \in X$.

Proof In Theorem 3.1, let $\varphi(x, y)=\theta\left(\|x\|^{p}\|y\|^{q}+\|x\|^{p+q}+\|y\|^{p+q}\right)$. Then we can choose $L=2^{j[(p+q)-2]}$ and we get the desired result.

Remark 4 Due to the condition (1), all results obtained in this section still hold true, even if the fuzzy set-valued mapping $f: X \rightarrow \mathcal{F}_{K C}(Y)$ degenerates into a set-valued mapping $f: X \rightarrow \mathcal{K}_{C}(Y)$ or a single-valued mapping $f: X \rightarrow Y$. Therefore, these results can be regarded as an important extension of the stability results of the ordinary single-valued and set-valued quadratic functional equations. In essence, our results extend some main results obtained by Cholewa [35], Czerwik [11] and Jung et al. [36].

\section{Hyers-Ulam stability of the Deeba type fuzzy set-valued functional equation}

In this section, we will consider the Hyers-Ulam stability of the Deeba type quadratic fuzzy set-valued functional equation by using the direct method. 
Definition 4.1 Let $X$ be a real vector space and let $f: X \rightarrow \mathcal{F}_{K C}(Y)$ be a fuzzy set-valued mapping. The Deeba type fuzzy set-valued functional equation is defined by

$$
f(x+y+z) \oplus f(x) \oplus f(y) \oplus f(z)=f(x+y) \oplus f(y+z) \oplus f(z+x)
$$

for all $x, y, z \in X$, where the equal sign means that the both sides of (10) (two fuzzy sets) are equal pointwise. Every solution of (10) is called a Deeba type fuzzy set-valued mapping.

Remark 5 In a similar way as shown before, it is easy to verify that the triangular fuzzy set-valued mapping $f$ constructed in Example 1 is a solution of (10). Similarly, $f(t)=t^{2} u_{0}$ is also a solution of (10) for any fixed fuzzy set $u_{0} \in \mathcal{F}_{K C}(Y)$. Moreover, these two examples are suitable for (23) which is introduced in the next section.

Lemma 4.1 Let $X$ be a real vector space. For a given $\epsilon \geq 0$, if a mapping $f: X \rightarrow \mathcal{F}_{K C}(Y)$ satisfies the following inequality:

$$
d_{\infty}(f(x+y+z) \oplus f(x) \oplus f(y) \oplus f(z), f(x+y) \oplus f(y+z) \oplus f(z+x)) \leq \epsilon
$$

for all $x, y, z \in X$, then

$$
d_{\infty}\left(f(x) \oplus \frac{2^{n}-1}{2^{2 n+1}} f\left(-2^{n} x\right), \frac{2^{n}+1}{2^{2 n+1}} f\left(2^{n} x\right)\right) \leq 3 \epsilon \sum_{k=1}^{n} 2^{-k}
$$

for all $x \in X$ and $n \in \mathbb{N}^{+}$.

Proof Letting $x=y=z=0$ in (11), we get $d_{\infty}\left(f(0), I_{0}\right) \leq \epsilon$. Putting $x=y=-z$ in (11), we get

$$
d_{\infty}(3 f(x) \oplus f(-x), 2 f(0) \oplus f(2 x)) \leq \epsilon .
$$

According to the properties of the supremum metric, we can obtain

$$
\begin{aligned}
& d_{\infty}(3 f(x) \oplus f(-x), f(2 x)) \\
& \quad \leq d_{\infty}(3 f(x) \oplus f(-x), 2 f(0) \oplus f(2 x))+d_{\infty}(2 f(0) \oplus f(2 x), f(2 x)) \\
& =d_{\infty}(3 f(x) \oplus f(-x), 2 f(0) \oplus f(2 x))+2 d_{\infty}\left(f(0), I_{0}\right) \\
& \leq 3 \epsilon .
\end{aligned}
$$

Furthermore, by substituting $-x$ for $x$ in (13), we have

$$
d_{\infty}(3 f(-x) \oplus f(x), f(-2 x)) \leq 3 \epsilon
$$

By (13) and (14), we can infer that

$$
\begin{aligned}
& d_{\infty}\left(f(x) \oplus \frac{1}{8} f(-2 x), \frac{3}{8} f(2 x)\right) \\
& =d_{\infty}\left(\frac{9}{8} f(x) \oplus \frac{1}{8} f(-2 x) \oplus \frac{3}{8} f(-x), \frac{3}{8} f(2 x) \oplus \frac{3}{8} f(-x) \oplus \frac{1}{8} f(x)\right)
\end{aligned}
$$




$$
\begin{aligned}
& \leq \frac{3}{8} d_{\infty}(3 f(x) \oplus f(-x), f(2 x))+\frac{1}{8} d_{\infty}(f(-2 x), 3 f(-x) \oplus f(x)) \\
& \leq \frac{9}{8} \epsilon+\frac{3}{8} \epsilon=\frac{3}{2} \epsilon,
\end{aligned}
$$

which implies that the inequality (12) holds true for $n=1$. By mathematical induction, we can assume that the inequality (12) is true for some $n \in \mathbb{N}^{+}$. Therefore, we have

$$
\begin{aligned}
d_{\infty}\left(f(x) \oplus \frac{2^{n+1}-1}{2^{2 n+3}} f\left(-2^{n+1} x\right), \frac{2^{n+1}+1}{2^{2 n+3}} f\left(2^{n+1} x\right)\right) \\
=d_{\infty}\left(f(x) \oplus \frac{2^{n}-1}{2^{2 n+1}} f\left(-2^{n} x\right) \oplus \frac{2^{n+1}+1}{2^{2 n+3}}\left(3 f\left(2^{n} x\right) \oplus f\left(-2^{n} x\right)\right)\right. \\
\quad \oplus \frac{2^{n+1}-1}{2^{2 n+3}} f\left(-2^{n+1} x\right), \frac{2^{n+1}+1}{2^{2 n+3}} f\left(2^{n+1} x\right) \oplus \frac{2^{n+1}-1}{2^{2 n+3}}\left(3 f\left(-2^{n} x\right) \oplus f\left(2^{n} x\right)\right) \\
\left.\quad \oplus \frac{2^{n}+1}{2^{2 n+1}} f\left(2^{n} x\right)\right) \\
\leq d_{\infty}\left(f(x) \oplus \frac{2^{n}-1}{2^{2 n+1}} f\left(-2^{n} x\right), \frac{2^{n}+1}{2^{2 n+1}} f\left(2^{n} x\right)\right)+\frac{2^{n+1}+1}{2^{2 n+3}} d_{\infty}\left(3 f\left(2^{n} x\right)\right. \\
\left.\quad \oplus f\left(-2^{n} x\right), f\left(2^{n+1} x\right)\right)+\frac{2^{n+1}-1}{2^{2 n+3}} d_{\infty}\left(3 f\left(-2^{n} x\right) \oplus f\left(2^{n} x\right), f\left(-2^{n+1} x\right)\right) \\
\leq 3 \epsilon\left(\sum_{k=1}^{n} 2^{-k}+\frac{2^{n+1}+1}{2^{2 n+3}}+\frac{2^{n+1}-1}{2^{2 n+3}}\right) \\
=3 \epsilon \sum_{k=1}^{n+1} 2^{-k},
\end{aligned}
$$

which proves the validity of the inequality (12) for $n+1$. This completes the proof.

Theorem 4.2 Let $X$ be a real vector space. Assume that a mapping $f: X \rightarrow \mathcal{F}_{K C}(Y)$ satisfies the inequality (11) and the following inequality:

$$
d_{\infty}(f(x), f(-x)) \leq \theta
$$

for some $\theta \geq 0$ and for all $x \in X$. Then there exists a unique Deeba quadratic fuzzy setvalued mapping $Q: X \rightarrow \mathcal{F}_{K C}(Y)$ such that

$$
d_{\infty}(f(x), Q(x)) \leq 3 \epsilon
$$

for all $x \in X$ with $Q(x)=Q(-x)$.

Proof According to Lemma 4.1 and the condition (15), we can infer that

$$
\begin{aligned}
& d_{\infty}\left(f(x), 2^{-2 n} f\left(2^{n} x\right)\right) \\
& \quad=d_{\infty}\left(f(x) \oplus \frac{2^{n}-1}{2^{2 n+1}} f\left(-2^{n} x\right), \frac{2^{n}-1}{2^{2 n+1}} f\left(-2^{n} x\right) \oplus 2^{-2 n} f\left(2^{n} x\right)\right) \\
& \quad \leq d_{\infty}\left(f(x) \oplus \frac{2^{n}-1}{2^{2 n+1}} f\left(-2^{n} x\right), \frac{2^{n}+1}{2^{2 n+1}} f\left(2^{n} x\right)\right)
\end{aligned}
$$




$$
\begin{aligned}
& +d_{\infty}\left(\frac{2^{n}+1}{2^{2 n+1}} f\left(2^{n} x\right), \frac{2^{n}-1}{2^{2 n+1}} f\left(-2^{n} x\right) \oplus 2^{-2 n} f\left(2^{n} x\right)\right) \\
\leq & 3 \epsilon \sum_{k=1}^{n} 2^{-k}+\frac{2^{n}-1}{2^{2 n+1}} d_{\infty}\left(f\left(2^{n} x\right), f\left(-2^{n} x\right)\right) \\
\leq & 3 \epsilon \sum_{k=1}^{n} 2^{-k}+\frac{2^{n}-1}{2^{2 n+1}} \theta .
\end{aligned}
$$

For $n \geq m$, by (17), we can obtain

$$
\begin{aligned}
& d_{\infty}\left(2^{-2 n} f\left(2^{n} x\right), 2^{-2 m} f\left(2^{m} x\right)\right) \\
& \quad=2^{-2 m} d_{\infty}\left(2^{-2(n-m)} f\left(2^{n-m} \cdot 2^{m} x\right), f\left(2^{m} x\right)\right) \\
& \quad \leq 2^{-2 m}\left(3 \epsilon \sum_{k=1}^{n-m} 2^{-k}+\frac{2^{n-m}-1}{2^{2(n-m)+1}} \theta\right) .
\end{aligned}
$$

It is easy to see that the sequence $\left\{2^{-2 n} f\left(2^{n} x\right)\right\}$ is Cauchy sequence, since the last expression of the inequality (18) tends to zero as $m \rightarrow \infty$. From the completeness of the metric space $\left(\mathcal{F}_{K C}(Y), d_{\infty}\right)$, the sequence $\left\{2^{-2 n} f\left(2^{n} x\right)\right\}$ is convergent for any $x \in X$. Set

$$
Q(x)=\lim _{n \rightarrow \infty} 2^{-2 n} f\left(2^{n} x\right) .
$$

To show that $Q$ satisfies (10), one can replace $x, y, z$ in (11) by $2^{n} x, 2^{n} y, 2^{n}, z$, respectively, it follows that

$$
\begin{gathered}
d_{\infty}\left(f\left(2^{n}(x+y+z)\right) \oplus f\left(2^{n} x\right) \oplus f\left(2^{n} y\right) \oplus f\left(2^{n} z\right), f\left(2^{n}(x+y)\right)\right. \\
\left.\oplus f\left(2^{n}(y+z)\right) \oplus f\left(2^{n}(z+x)\right)\right) \leq \epsilon .
\end{gathered}
$$

Dividing both sides by $2^{2 n}$ in the last inequality, and by letting $n \rightarrow \infty$, it is easy to see that $Q$ is a solution of (10). In a similar manner, we can find the condition (15) implies that $Q(x)=Q(-x)$. Moreover, by letting $n \rightarrow \infty$ in (17), it can easily be verified that the inequality (16) holds true.

Now we show the uniqueness of $Q$. Let $Q^{\prime}$ be another Deeba quadratic mapping which satisfies the equality (10) and the inequality (16). It is obvious that $Q\left(2^{n} x\right)=4^{n} Q(x)$ and $Q^{\prime}\left(2^{n} x\right)=4^{n} Q^{\prime}(x)$ for all $x \in X$ and $n \in \mathbb{N}$. Then it follows from (16) that

$$
\begin{aligned}
d_{\infty}\left(Q(x), Q^{\prime}(x)\right) & =4^{-n} d_{\infty}\left(Q\left(2^{n} x\right), Q^{\prime}\left(2^{n} x\right)\right) \\
& \leq 4^{-n}\left(d_{\infty}\left(Q\left(2^{n} x\right), f\left(2^{n} x\right)\right)+d_{\infty}\left(f\left(2^{n} x\right), Q^{\prime}\left(2^{n} x\right)\right)\right) \\
& \leq \frac{6 \epsilon}{4^{n}}
\end{aligned}
$$

for all $x \in X$. By letting $n \rightarrow \infty$, we conclude that $Q(x) \equiv Q^{\prime}(x)$. The proof of the theorem is now completed.

Theorem 4.3 Let X be a real vector space. Assume that a mapping $: X \rightarrow \mathcal{F}_{K C}(Y)$ satisfies the inequality (11) and the following inequality:

$$
d_{\infty}\left(f(x) \oplus f(-x), I_{0}\right) \leq \theta
$$


for some $\theta \geq 0$ and for all $x \in X$. Then there exists a unique Deeba type additive fuzzy set-valued mapping $A: X \rightarrow \mathcal{F}_{K C}(Y)$ such that

$$
d_{\infty}(f(x), A(x)) \leq 3 \epsilon
$$

for all $x \in X$ with $A(x) \oplus A(-x)=I_{0}$.

Proof By Lemma 4.1 and the condition (15), we have

$$
\begin{aligned}
& d_{\infty}\left(f(x), 2^{-n} f\left(2^{n} x\right)\right) \\
& =d_{\infty}\left(f(x) \oplus \frac{2^{n}-1}{2^{2 n+1}} f\left(-2^{n} x\right), \frac{2^{n}-1}{2^{2 n+1}} f\left(-2^{n} x\right) \oplus 2^{-2 n} f\left(2^{n} x\right)\right) \\
& \leq d_{\infty}\left(f(x) \oplus \frac{2^{n}-1}{2^{2 n+1}} f\left(-2^{n} x\right), \frac{2^{n}+1}{2^{2 n+1}} f\left(2^{n} x\right)\right) \\
& \quad+d_{\infty}\left(\frac{2^{n}+1}{2^{2 n+1}} f\left(2^{n} x\right), \frac{2^{n}-1}{2^{2 n+1}} f\left(-2^{n} x\right) \oplus 2^{-2 n} f\left(2^{n} x\right)\right) \\
& \leq 3 \epsilon \sum_{k=1}^{n} 2^{-k}+\frac{2^{n}-1}{2^{2 n+1}} d_{\infty}\left(I_{0}, f\left(2^{n} x\right) \oplus f\left(-2^{n} x\right)\right) \\
& \leq 3 \epsilon \sum_{k=1}^{n} 2^{-k}+\frac{2^{n}-1}{2^{2 n+1}} \theta .
\end{aligned}
$$

By the preceding inequality, for $n \geq m$, we can obtain

$$
\begin{aligned}
& d_{\infty}\left(2^{-n} f\left(2^{n} x\right), 2^{-m} f\left(2^{m} x\right)\right) \\
& \quad=2^{-m} d_{\infty}\left(2^{-(n-m)} f\left(2^{n-m} \cdot 2^{m} x\right), f\left(2^{m} x\right)\right) \\
& \quad \leq 2^{-m}\left(3 \epsilon \sum_{k=1}^{n-m} 2^{-k}+\frac{2^{n-m}-1}{2^{2(n-m)+1}} \theta\right)
\end{aligned}
$$

which means that the sequence $\left\{2^{-n} f\left(2^{n} x\right)\right\}$ is a Cauchy sequence in the complete metric space $\left(\mathcal{F}_{K C}(Y), d_{\infty}\right)$. Therefore, we can define

$$
A(x)=\lim _{n \rightarrow \infty} 2^{-n} f\left(2^{n} x\right)
$$

for all $x \in X$. Using the same argument as in the proof of Theorem 4.2, we conclude that the mapping $A$ satisfies the equality (10) with $A(x) \oplus A(-x)=I_{0}$. Clearly, we get $A(0)=I_{0}$.

Putting $z=-y$ in (10). According to the preceding properties of $A$, let $u=x+y, v=x-y$, we can obtain

$$
2 A\left(\frac{u+v}{2}\right)=A(u)+A(v) .
$$

That is to say, the mapping $A$ is additive fuzzy set-valued mapping due to [37].

We now show that $A$ is a unique Deeba type additive mapping. Let $A^{\prime}$ be another mapping which satisfies the equality (10) and the inequality (20). Since $A\left(2^{n} x\right)=2^{n} A(x)$, 


$$
\begin{aligned}
& A^{\prime}\left(2^{n} x\right)=2^{n} A^{\prime}(x), \text { we get } \\
& \begin{aligned}
d_{\infty}\left(A(x), A^{\prime}(x)\right) & =2^{-n} d_{\infty}\left(A\left(2^{n} x\right), A^{\prime}\left(2^{n} x\right)\right) \\
& \leq 2^{-n}\left(d_{\infty}\left(A\left(2^{n} x\right), f\left(2^{n} x\right)\right)+d_{\infty}\left(f\left(2^{n} x\right), A^{\prime}\left(2^{n} x\right)\right)\right) \\
& \leq \frac{6 \epsilon}{2^{n}}
\end{aligned}
\end{aligned}
$$

for all $x \in X$. By letting $n \rightarrow \infty$, we conclude that $A(x) \equiv A^{\prime}(x)$. This completes the proof.

Remark 6 From Theorems 4.2 and 4.3, the condition (15) ensures that the mapping is quadratic, whereas the condition (19) implies that the mapping is additive.

Remark 7 The preceding theorems extend some stability results of the single-valued Deeba type functional equation obtained by Jung [13].

\section{Hyers-Ulam stability of the Appolonius type quadratic fuzzy set-valued functional equation}

Here we shall prove the Hyers-Ulam stability of the Appolonius type quadratic fuzzy setvalued functional equation by using the same method as employed in the preceding section.

Definition 5.1 Let $X$ be a real vector space and let $f: X \rightarrow \mathcal{F}_{K C}(Y)$ be a fuzzy set-valued mapping. The Appolonius type fuzzy set-valued functional equation is defined by

$$
f(x-z) \oplus f(y-z)=\frac{1}{2} f(x-y) \oplus 2 f\left(z-\frac{x+y}{2}\right)
$$

for all $x, y, z \in X$, where the equal sign means that the both sides of (23) (two fuzzy sets) are equal pointwise. Every solution of (23) is called an Appolonius type fuzzy set-valued mapping.

Theorem 5.1 Let $j \in\{-1,1\}$ be fixed and let $\varphi: X \times X \times X \rightarrow[0, \infty)$ be a function such that

$$
\Phi^{(j)}(x, y, z)=\sum_{k=0}^{\infty} 4^{-j k} \varphi\left(2^{j k} x, 2^{j k} y, 2^{j k} z\right)<+\infty
$$

for all $x, y, z \in X$. Assume that the mapping $f: X \rightarrow \mathcal{F}_{K C}(Y)$ satisfies

$$
d_{\infty}\left(f(x-z) \oplus f(y-z), \frac{1}{2} f(x-y) \oplus 2 f\left(z-\frac{x+y}{2}\right)\right) \leq \varphi(x, y, z)
$$

for all $x, y, z \in X$. Moreover, $f(0)=I_{0}$ in the case $j=1$. Then

$$
Q(x)=\lim _{n \rightarrow \infty} 4^{-j n} f\left(2^{j n} x\right)
$$


exists for each $x \in X$ and defines a unique Appolonius type quadratic fuzzy set-valued mapping $Q: X \rightarrow \mathcal{F}_{K C}(Y)$ such that

$$
d_{\infty}(f(x), Q(x)) \leq \frac{1}{2} \Phi^{(j)}(x,-x, x)+\frac{1}{4} \Phi^{(j)}(x, x,-x)
$$

for all $x \in X$.

Proof Firstly, we claim that $f(0)=I_{0}$ in the case $j=-1$. Indeed, letting $x=y=z=0$ in (25), we can infer that $f(0)=I_{0}$ because the condition $\Phi(0,0,0)=\sum_{k=0}^{\infty} 4^{k} \varphi(0,0,0)<+\infty$ implies that $\varphi(0,0,0)=0$. So we have $f(0)=I_{0}$ regardless of $j=1$ or $j=-1$.

Replacing $y, z$ by $x,-x$ in (25), respectively, we have

$$
d_{\infty}(2 f(2 x), f(-2 x)) \leq \varphi(x, x,-x)
$$

for all $x \in X$. Substituting $y, z$ by $-x, x$ in (25), respectively, we get

$$
d_{\infty}\left(f(-2 x), \frac{1}{2} f(2 x) \oplus 2 f(x)\right) \leq \varphi(x,-x, x)
$$

for all $x \in X$. From the inequalities (27) and (28), it follows that

$$
\begin{aligned}
d_{\infty}(f(2 x), 4 f(x)) & =d_{\infty}(4 f(x) \oplus f(2 x), 2 f(2 x)) \\
& \leq d_{\infty}(4 f(x) \oplus f(2 x), 2 f(-2 x))+d_{\infty}(f(-2 x), 2 f(2 x)) \\
& \leq \varphi(x, x,-x)+2 \varphi(x,-x, x)
\end{aligned}
$$

for all $x \in X$. Dividing both sides in (29) by 4 , we obtain

$$
d_{\infty}\left(\frac{1}{4} f(2 x), f(x)\right) \leq \frac{1}{4} \varphi(x, x,-x)+\frac{1}{2} \varphi(x,-x, x)
$$

for all $x \in X$. Replacing $x$ by $2^{n-1} x$ and dividing by $4^{n-1}$ in (30), we conclude that

$$
\begin{aligned}
& d_{\infty}\left(\frac{1}{4^{n}} f\left(2^{n} x\right), \frac{1}{4^{n-1}} f\left(2^{n-1} x\right)\right) \\
& \quad \leq \frac{\varphi\left(2^{n-1} x, 2^{n-1} x,-2^{n-1} x\right)}{4^{n}}+\frac{\varphi\left(2^{n-1} x,-2^{n-1} x, 2^{n-1} x\right)}{2 \cdot 4^{n-1}}
\end{aligned}
$$

for all $x \in X$ and for all $n \in \mathbb{N}$. Combining the inequalities (30) and (31) gives

$$
d_{\infty}\left(f(x), 4^{-n} f\left(2^{n} x\right)\right) \leq \sum_{i=0}^{n-1} \frac{\varphi\left(2^{i} x,-2^{i} x, 2^{i} x\right)}{2 \cdot 4^{i}}+\sum_{i=0}^{n-1} \frac{\varphi\left(2^{i} x, 2^{i} x,-2^{i} x\right)}{4^{i+1}}
$$

for all $x \in X$ and for all $n \in \mathbb{N}$.

Moreover, substituting $x$ by $\frac{x}{2}$ in (29), we get

$$
d_{\infty}\left(f(x), 4 f\left(\frac{x}{2}\right)\right) \leq \varphi\left(\frac{x}{2}, \frac{x}{2},-\frac{x}{2}\right)+2 \varphi\left(\frac{x}{2},-\frac{x}{2}, \frac{x}{2}\right)
$$


for all $x \in X$. By using a similar argument, we can infer that

$$
d_{\infty}\left(f(x), 4^{n} f\left(\frac{x}{2^{n}}\right)\right) \leq \frac{1}{4} \sum_{i=1}^{n} 4^{i} \varphi\left(\frac{x}{2^{i}}, \frac{x}{2^{i}},-\frac{x}{2^{i}}\right)+\frac{1}{2} \sum_{i=1}^{n} 4^{i} \varphi\left(\frac{x}{2^{i}},-\frac{x}{2^{i}}, \frac{x}{2^{i}}\right)
$$

for all $x \in X$ and $n \in \mathbb{N}$. Here we claim that the sequence $\left\{4^{-j n} f\left(2^{j n} x\right)\right\}$ is a Cauchy sequence in the complete metric space $\left(\mathcal{F}_{K C}(Y), d_{\infty}\right)$. Indeed, it follows from (32) and (34) that

$$
\begin{aligned}
& d_{\infty}\left(4^{-j(n+m)} f\left(2^{j(n+m)} x\right), 4^{-j m} f\left(2^{j m} x\right)\right) \\
& \quad=4^{-j m} d_{\infty}\left(4^{-j n} f\left(2^{j n} \cdot 2^{j m} x\right), f\left(2^{j m} x\right)\right) \\
& \quad \leq \begin{cases}\frac{1}{4^{m}}\left(\sum_{i=0}^{n-1} \frac{\varphi\left(2^{i+m} x,-2^{i+m} x, 2^{i+m} x\right)}{2 \cdot 4^{i}}+\sum_{i=0}^{n-1} \frac{\varphi\left(2^{i+m} x, 2^{i+m} x,-2^{i+m} x\right)}{4^{i+1}}\right), & j=1, \\
\frac{1}{4} \sum_{i=1}^{n} 4^{i+m} \varphi\left(\frac{x}{2^{i+m}}, \frac{x}{2^{i+m}},-\frac{x}{2^{i+m}}\right)+\frac{1}{2} \sum_{i=1}^{n} 4^{i+m+1} \varphi\left(\frac{x}{2^{i+m}},-\frac{x}{2^{i+m}}, \frac{x}{2^{i}}\right), & j=-1\end{cases}
\end{aligned}
$$

for all $x \in X$ and for all $n, m \in \mathbb{N}$. When $j=1$, it is obvious that the last expression tends to zero as $m \rightarrow \infty$. When $j=-1$, we can infer from the condition (24) that the preceding expression also tends to zero as $m \rightarrow \infty$. Thus, we have proved that the sequence $\left\{4^{-j n} f\left(2^{j n} x\right)\right\}$ is Cauchy. By the completeness of $\mathcal{F}_{K C}(Y)$, we can define

$$
Q(x)=\lim _{n \rightarrow \infty} 4^{-j n} f\left(2^{j n} x\right)
$$

for all $x \in X$.

Now we show that $Q$ satisfies (23). Replacing $x, y, z$ in (25) by $2^{j n} x, 2^{j n} y, 2^{j n} z$, respectively, and dividing both sides by $4^{\text {jn }}$, we can obtain

$$
\begin{aligned}
& 4^{-j n} d_{\infty}\left(f\left(2^{j n}(x-z)\right) \oplus f\left(2^{j n}(y-z)\right), \frac{1}{2} f\left(2^{j n}(x-y)\right) \oplus 2 f\left(2^{j n}\left(z-\frac{x+y}{2}\right)\right)\right) \\
& \quad \leq 4^{-j n} \varphi\left(2^{j n} x, 2^{j n} y, 2^{j n} z\right) .
\end{aligned}
$$

Taking the limit in the preceding expression, it can easily be seen that $Q$ satisfies (23) since the right hand side tends to zero as $n \rightarrow \infty$.

By letting $n \rightarrow \infty$ in (32) and (34), we can infer that

$$
d_{\infty}(f(x), Q(x)) \leq \begin{cases}\frac{1}{2} \Phi^{(1)}(x,-x, x)+\frac{1}{4} \Phi^{(1)}(x, x,-x), & j=1, \\ \frac{1}{2} \Phi^{(-1)}(x,-x, x)+\frac{1}{4} \Phi^{(-1)}(x, x,-x), & j=-1\end{cases}
$$

for all $x \in X$.

Let us prove the uniqueness. Let $Q^{\prime}$ be another Appolonius type quadratic fuzzy setvalued mapping satisfying the inequality (26). Since $Q\left(2^{j n} x\right)=4^{j n} Q(x), Q^{\prime}\left(2^{j n} x\right)=4^{j n} Q^{\prime}(x)$, we have

$$
\begin{aligned}
d_{\infty}\left(Q(x), Q^{\prime}(x)\right) & =4^{-j n} d_{\infty}\left(Q\left(2^{j n} x\right), Q^{\prime}\left(2^{j n} x\right)\right) \\
& \leq 4^{-j n}\left(d_{\infty}\left(Q\left(2^{j n} x\right), f\left(2^{j n} x\right)\right)+d_{\infty}\left(f\left(2^{j n} x\right), Q^{\prime}\left(2^{j n} x\right)\right)\right) \\
& \leq 4^{-j n}\left(\frac{1}{2} \Phi^{(j)}\left(2^{j n} x,-2^{j n} x, 2^{j n} x\right)+\frac{1}{4} \Phi^{(j)}\left(2^{j n} x, 2^{j n} x,-2^{j n} x\right)\right) .
\end{aligned}
$$


From the condition (24), it follows that the last expression tends to zero as $n \rightarrow \infty$. Thus, we conclude that $Q(x) \equiv Q^{\prime}(x)$. This completes the proof.

Based on Theorem 5.1, we can obtain the following corollaries.

Corollary 5.2 Let $j \in\{-1,1\}$ be fixed. Suppose that $X$ is a real normed space and $p, \theta$ are positive real numbers with $p \neq 2$. Assume that the mapping $f: X \rightarrow \mathcal{F}_{K C}(Y)$ satisfies

$$
d_{\infty}\left(f(x-z) \oplus f(y-z), \frac{1}{2} f(x-y) \oplus 2 f\left(z-\frac{x+y}{2}\right)\right) \leq \theta\left(\|x\|^{p}+\|y\|^{p}+\|z\|^{p}\right)
$$

for all $x, y \in X$. Moreover, $f(0)=I_{0}$ in the case $j=1$. Then

$$
Q(x)=\lim _{n \rightarrow \infty} 4^{-j n} f\left(2^{j n} x\right)
$$

exists for each $x \in X$ and defines a unique Appolonius type quadratic fuzzy set-valued mapping $Q: X \rightarrow \mathcal{F}_{K C}(Y)$ such that

$$
d_{\infty}(f(x), Q(x)) \leq \begin{cases}\frac{9 \theta\|x\|^{p}}{4-2^{p}}, & \text { if } j=1, p<2, \\ \frac{9 \theta\|x\|^{p}}{4-2^{4-p},}, & \text { if } j=-1, p>2\end{cases}
$$

for all $x \in X$.

Remark 8 In Theorem 5.1 and Corollary 5.2, if the fuzzy set-valued mapping $f$ degenerates into a single-valued mapping, then the supremum metric $d_{\infty}$ will reduce to the ordinary metric between two points. Thus, these results can be viewed as an extension of the main results obtained by Jun and Kim [14].

Corollary 5.3 Let $j \in\{-1,1\}$ be fixed. Suppose that $X$ is a real normed space and $p, \theta$ are positive real numbers with $p \neq \frac{2}{3}$. Assume that the mapping $f: X \rightarrow \mathcal{F}_{K C}(Y)$ satisfies

$$
d_{\infty}\left(f(x-z) \oplus f(y-z), \frac{1}{2} f(x-y) \oplus 2 f\left(z-\frac{x+y}{2}\right)\right) \leq \theta\|x\|^{p}\|y\|^{p}\|z\|^{p}
$$

for all $x, y \in X$. Moreover, $f(0)=I_{0}$ in the case $j=1$. Then

$$
Q(x)=\lim _{n \rightarrow \infty} 4^{-j n} f\left(2^{j n} x\right)
$$

exists for each $x \in X$ and defines a unique Appolonius type quadratic fuzzy set-valued mapping $Q: X \rightarrow \mathcal{F}_{K C}(Y)$ such that

$$
d_{\infty}(f(x), Q(x)) \leq \begin{cases}\frac{3 \theta\|x\|^{3 p}}{4-2^{3 p}}, & \text { if } j=1, p<\frac{2}{3}, \\ \frac{3 \theta\|x\|^{3 p}}{4-2^{4-3 p}}, & \text { if } j=-1, p>\frac{2}{3}\end{cases}
$$

for all $x \in X$.

Corollary 5.4 Let $j \in\{-1,1\}$ be fixed. Suppose that $X$ is a real normed space and $p, q, r$, $\theta$ are positive real numbers with $p+q+r \neq 2$. Assume that the mapping $f: X \rightarrow \mathcal{F}_{K C}(Y)$ 
satisfies

$$
\begin{aligned}
& d_{\infty}\left(f(x-z) \oplus f(y-z), \frac{1}{2} f(x-y) \oplus 2 f\left(z-\frac{x+y}{2}\right)\right) \\
& \quad \leq \theta\left(\|x\|^{p}\|y\|^{q}\|z\|^{r}+\|x\|^{p+q+r}+\|y\|^{p+q+r}+\|z\|^{p+q+r}\right)
\end{aligned}
$$

for all $x, y \in X$. Moreover, $f(0)=I_{0}$ in the case $j=1$. Then

$$
Q(x)=\lim _{n \rightarrow \infty} 4^{-j n} f\left(2^{j n} x\right)
$$

exists for each $x \in X$ and defines a unique Appolonius type quadratic fuzzy set-valued mapping $Q: X \rightarrow \mathcal{F}_{K C}(Y)$ such that

$$
d_{\infty}(f(x), Q(x)) \leq \begin{cases}\frac{3 \theta\|x\| \|^{p+q+r}}{1-2^{(p+q+r)-2},}, & \text { if } j=1, p+q+r<2, \\ \frac{3 \theta\|x\|^{p+q+r}}{1-2^{(p+q+r)-2}}, & \text { if } j=-1, p+q+r>2\end{cases}
$$

for all $x \in X$.

Corollary 5.5 Let $X$ is a real normed space. For a given $\theta>0$, if the mapping $f: X \rightarrow$ $\mathcal{F}_{K C}(Y)$ satisfies

$$
d_{\infty}\left(f(x-z) \oplus f(y-z), \frac{1}{2} f(x-y) \oplus 2 f\left(z-\frac{x+y}{2}\right)\right) \leq \theta
$$

for all $x, y \in X$ and with $f(0)=I_{0}$, then

$$
Q(x)=\lim _{n \rightarrow \infty} 4^{-n} f\left(2^{n} x\right)
$$

exists for each $x \in X$ and defines a unique Appolonius type quadratic fuzzy set-valued mapping $Q: X \rightarrow \mathcal{F}_{K C}(Y)$ such that

$$
d_{\infty}(f(x), Q(x)) \leq \theta
$$

for all $x \in X$.

\section{Competing interests}

The authors declare that they have no competing interests.

\section{Authors? contributions}

All authors contributed equally and significantly in writing this article. All authors read and approved the final manuscript.

\section{Author details}

'School of Mathematics and Statistics, Tianshui Normal University, Tianshui, 741001, P.R. China. ${ }^{2}$ School of Mathematics and Statistics, Beijing Institute of Technology, Beijing, 100081, P.R. China. ${ }^{3}$ School of Information, Capital University of Economics and Business, Beijing, 100070, P.R. China. ${ }^{4}$ Department of Mathematics and Finance, Chongqing University of Arts and Sciences, Yongchuan, 402160, P.R. China. 


\section{Acknowledgements}

This work was supported by ? Qing Lan? Talent Engineering Funds by Tianshui Normal University, and the Research Project of Higher Learning of Gansu Province (No. 2014B-080). The second author acknowledges the support of the Humanity and Social Science Youth Foundation of Ministry of Education of China (No. 13YJC630012), and the Specially Commissioned Project of the Capital University of Economics and Business. The third author acknowledges the support of the National Natural Science Foundation of China (No. 11226268).

Received: 27 September 2014 Accepted: 13 December 2014 Published online: 09 January 2015

\section{References}

1. Ulam, SM: Problems in Modern Mathematics. Wiley, New York (1960)

2. Hyers, DH: On the stability of the linear functional equation. Proc. Natl. Acad. Sci. USA 27, $222-224$ (1941)

3. Aoki, T: On the stability of the linear transformation in Banach spaces. J. Math. Soc. Jpn. 2, 64-66 (1950)

4. Rassias, TM: On the stability of the linear mapping in Banach spaces. Proc. Am. Math. Soc. 72, 297-300 (1978)

5. Găvruța, P: A generalization of the Hyers-Ulam-Rassias stability of approximately additive mappings. J. Math. Anal. Appl. 184, 431-436 (1994)

6. Cho, YJ, Rassias, TM, Saadati, R: Stability of Functional Equations in Random Normed Spaces. Springer, New York (2013)

7. Forti, GL: Hyers-Ulam stability of functional equations in several variables. Aequ. Math. 50, 143-190 (1995)

8. Jung, SM: Hyers-Ulam-Rassias Stability of Functional Equations in Nonlinear Analysis. Springer, New York (2011)

9. Kannappan, P: Functional Equations and Inequalities with Applications. Springer, New York (2009)

10. Rassias, TM: On the stability of functional equations and a problem of Ulam. Acta Appl. Math. 62, 23-130 (2000)

11. Czerwik, S: On the stability of the quadratic mapping in normed spaces. Abh. Math. Semin. Univ. Hamb. 62, 59-64 (1992)

12. Kim, GH: On the stability of the quadratic mapping in normed spaces. Int. J. Math. Math. Sci. 25, 217-229 (2001)

13. Jung, SM: On the Hyers-Ulam-Rassias stability of the functional equations that have quadratic property. J. Math. Anal. Appl. 222, 126-137 (1998)

14. Jun, KW, Kim, HM: On the stability of Appolonius? equation. Bull. Belg. Math. Soc. Simon Stevin11, 615-624 (2004)

15. Mirmostafaee, AK, Moslehian, MS: Fuzzy versions of Hyers-Ulam-Rassias theorem. Fuzzy Sets Syst. 159, $720-729$ (2008)

16. Mirmostafaee, AK, Mirzavaziri, M, Moslehian, MS: Fuzzy stability of the Jensen functional equation. Fuzzy Sets Syst. $159,730-738(2008)$

17. Abbaszadeh, S: Intuitionistic fuzzy stability of a quadratic and quartic functional equation. Int. J. Nonlinear Anal. Appl. $1,100-124(2010)$

18. Gordji, ME, Khodaei, H, Kamyar, M: Stability of Cauchy-Jensen type functional equation in generalized fuzzy normed spaces. Comput. Math. Appl. 62, 2950-2960 (2011)

19. Khodaei, H, Kamyar, M: Fuzzy approximately additive mappings. Int. J. Nonlinear Anal. Appl. 1, $44-53$ (2010)

20. Miheț, D: The fixed point method for fuzzy stability of the Jensen functional equation. Fuzzy Sets Syst. 160 1663-1667 (2009)

21. Miheț, D: The stability of the additive Cauchy functional equation in non-Archimedean fuzzy normed spaces. Fuzzy Sets Syst. 161, 2206-2212 (2010)

22. Mirmostafaee, AK: A fixed point approach to almost quartic mappings in quasi fuzzy normed spaces. Fuzzy Sets Syst. $160,1653-1662$ (2009)

23. Mohiuddine, SA, Alotaibi, A: Fuzzy stability of a cubic functional equation via fixed point technique. Adv. Differ. Equ. 2012, 48 (2012)

24. Shakeri, S, Saadati, R, Park, C: Stability of the quadratic functional equation in non-Archimedean $\mathcal{L}$-fuzzy normed spaces. Int. J. Nonlinear Anal. Appl. 1, 72-83 (2010)

25. Eskandani, GZ, Găvruţa, P, Kim, GH: On the stability problem in fuzzy Banach space. Abstr. Appl. Anal. 2012, Article ID $763728(2012)$

26. Lu, G, Park, C: Hyers-Ulam stability of additive set-valued functional equations. Appl. Math. Lett. 24, 1312-1316 (2011)

27. Park, C, O? Regan, D, Saadati, R: Stability of some set-valued functional equations. Appl. Math. Lett24, 1910-1914 (2011)

28. Kenary, HA, Rezaei, H, Gheisari, Y, Park, C: On the stability of set-valued functional equations with the fixed point alternative. Fixed Point Theory Appl. 2012,81 (2012)

29. Castaing, C, Valadier, M: Convex Analysis and Measurable Multifunctions. Lect. Notes Math., vol. 580. Springer, Berlin (1977)

30. Diamond, P, Kloeden, P: Metric Spaces of Fuzzy Sets: Theory and Applications. World Scientific, Singapore (1994)

31. Inoue, H: A strong law of large numbers for fuzzy random sets. Fuzzy Sets Syst. 41, 285-291 (1991)

32. Nikodem, K: K-Convex and K-Concave Set-Valued Functions. Zeszyty Nauk. Politech., Lodz (1989)

33. Diaz, JB, Margolis, B: A fixed point theorem of the alternative for contractions on a generalized complete metric space. Bull. Am. Math. Soc. 74, 305-309 (1968)

34. Gordji, ME, Park, C, Savadkouhi, MB: The stability of a quartic type functional equation with the fixed point alternative. Fixed Point Theory 11, 265-272 (2010)

35. Cholewa, PW: Remarks on the stability of functional equations. Aequ. Math. 27, 76-86 (1984)

36. Jung, SM, Kim, B, Lee, KS: A fixed point approach to the stability of quadratic functional equation. Bull. Korean Math. Soc. 43, 341-351 (2006)

37. Shen, YH, Lan, YY, Chen, W: Hyers-Ulam-Rassias stability of some additive fuzzy set-valued functional equations with the fixed point alternative. Abstr. Appl. Anal. 2014, Article ID 139175 (2014) 